\title{
Des interventions en français réinvesties dans la résolution de problèmes en mathématiques au primaire : Vers le développement d'un contrôle interdisciplinaire chez les élèves
}

\author{
Mireille Saboya \\ Université du Québec à Montréal \\ Mélanie Tremblay \\ Université du Québec à Rimouski
}

\section{Résumé}

La résolution de problèmes est un incontournable dans l'enseignement et l'apprentissage des mathématiques ; elle pose toutefois de nombreux défis aux élèves. Nous avons mené deux projets de recherche collaborative impliquant divers intervenants du milieu qui visaient à coconstruire des interventions favorisant le développement d'un contrôle chez les élèves lors de la résolution de problèmes en mathématiques au primaire. L'analyse des discussions au sein des rencontres réflexives révèle trois interventions des enseignants teintées par leur 
travail mené en français. Cet article présente le réinvestissement en mathématiques de ce qui est fait en français. Il aborde une réflexion quant aux liens qui pourraient unir différentes interventions entre ces deux disciplines et qui visent le développement d'un contrôle interdisciplinaire chez les élèves.

Mots-clés : interdisciplinarité, résolution de problèmes, mathématiques, français, contrôle

\begin{abstract}
Problem solving is an essential part of teaching and learning mathematics, but it poses many challenges for students. We conducted two collaborative research projects involving various educators to co-construct interventions that promote the development of student control during problem solving in elementary mathematics. The analysis of the discussions during the meetings revealed three interventions by the teachers that were influenced by their work in French. This paper presents this reinvestment in mathematics of what is done in French, and discusses possible links between these two disciplines in order to develop students' interdisciplinary control.
\end{abstract}

Keywords: interdisciplinarity, problem solving, mathematics teaching, French teaching, control 


\section{Contexte de l'étude}

L'enseignement et l'apprentissage de la résolution de problèmes est un enjeu important en mathématiques tant à l'ordre primaire que secondaire, et ce, depuis le début du XXe siècle au Québec (Lajoie et Bednarz, 2012, 2016). Cette thématique, apparaissant centrale dans de nombreux pays, a fait l'objet de plusieurs congrès (Freiman et Savard, 2014). Selon notre définition, la résolution d'un problème implique de la part de l'élève la mobilisation d'un contrôle qui se traduit par une réflexion sur tout choix ou toute action tout au long de la résolution, sur la capacité à prendre des décisions de façon réfléchie et une certaine prise de distance par rapport à la résolution (Saboya, 2010). Le contrôle exercé en résolution de problèmes a fait l'objet de plusieurs études : en algèbre (Morand, 2020 ; Saboya, 2010 ; Saboya et al., 2015), autour de la comparaison de fractions (Saboya et Rhéaume, 2015), autour du calcul différentiel (Dufour et Jeannotte, 2013) et pour des problèmes proportionnels (Rhéaume, 2020). Par ailleurs, l'enseignant joue un rôle important lors de la résolution de problèmes pour développer un contrôle chez les élèves (Bolduc, 2020 ; Saboya et Tremblay, 2017). Il est un « questionneur » amenant les élèves à réfléchir sur leurs actions (Fagnant et al., 2016 ; Ge et Land, 2003 ; Giroux, 2004 ; Kemmerle, 2013 ; Mottier Lopez, 2007). De plus, il permet le développement de leur pensée critique en les encourageant à exprimer leur point de vue, à le comparer à celui de leurs pairs et à juger de l'efficacité des solutions partagées (Tremblay et Dumas, 2011).

Intéressées à étudier les interventions pouvant favoriser un contrôle chez les élèves en adaptation scolaire, nous avons mené une recherche collaborative avec deux enseignantes, l'une intervenant en deuxième année et l'autre en troisième année au primaire. L'analyse des rencontres réflexives a permis de relever, dans les propos des enseignantes, des suggestions d'interventions en mathématiques teintées de l'enseignement qu'elles font en français ${ }^{1}$. Ce résultat a guidé notre regard lors de l'analyse des rencontres réflexives d'une autre recherche collaborative impliquant des enseignants et un conseiller pédagogique intervenant au troisième cycle du primaire autour du même objet de recherche. Dans cet article, nous proposons de vous présenter trois des interventions qui ont été dégagées et qui visent le déploiement d'un contrôle

1 Nous proposons, dans Saboya et Tremblay (2019), quelques-unes de ces interventions qui s'ajoutent à celles présentées dans ce texte. 
interdisciplinaire chez les élèves en résolution de problèmes. Ce travail entre le français et les mathématiques s'inscrit dans l'orientation du programme d'enseignement québécois. Il mise sur le décloisonnement disciplinaire, tant au niveau primaire (MEQ, 2000) que secondaire (MEQ, 2006).

Plusieurs recherches (Cummins et al., 1988 ; Devidal et al., 1997 ; Fayol, 1992 ; Reusser, 1990 ; Schleppegrell, 2007 ; Søvik et al., 1999 ; Vilenius-Tuohimaa et al., 2008) établissent un lien entre la compréhension d'un texte et les habiletés en résolution de problèmes. Ainsi, les difficultés en résolution de problèmes pourraient s'expliquer par le fait de ne pas être un bon lecteur (Devidal et al., 1997 ; Reusser, 1990), par l'usage de certains mots qui posent problème (Cummins et al., 1988), ou par la complexité du vocabulaire en mathématiques, qui comprend non seulement des termes spécifiques à ce domaine, mais également des termes tirés du langage de la vie quotidienne qui, utilisés en mathématiques, ont un sens différent du sens usuel (Schleppegrell, 2007). Pour Fayol (1992), comprendre un texte implique de se forger un modèle mental de son contenu en y faisant intervenir des connaissances de différents types : l'élève construit une représentation mentale nommée « modèle de situation » (Luquette, 2017). De leur côté, Duval (1991) et Radford (1996) contestent la réduction de la compréhension d'un texte en mathématiques à la compréhension de ce que le texte énonce littéralement. Ces chercheurs soulignent que la compréhension d'un problème mathématique n'est pas indépendante de sa résolution; elle s'affine et se modifie tout au long de la démarche de résolution. D'autres recherches mettent de l'avant une habileté en lecture reliée au rendement en résolution de problèmes : l'habileté à produire des inférences tant à l'ordre primaire (Goulet, 2013 ; Goulet et Voyer, 2014 ; Voyer et al., 2012) que secondaire (Luquette, 2017).

Ainsi, ces travaux s'attardent à circonscrire les similitudes et les différences associées à la compréhension de textes en français et à la résolution de problèmes en mathématiques. Le présent article s'écarte toutefois de ce qui précède au sens où il ne se tourne pas vers la comparaison des capacités des élèves, mais s'intéresse plutôt à la pratique enseignante en mathématiques afin de dégager le réinvestissement d'interventions en français pour les mathématiques. 


\section{Le concept de contrôle comme levier théorique}

Exercer du contrôle dans la résolution de problèmes implique une certaine manière d'aborder un problème mathématique, et ce, tout le long de la résolution, en amont, en cours et à la fin. Différentes composantes sont associées au contrôle, comme l'anticipation, la vérification, la perception d'erreurs, la mise en place d'un contrôle sémantique et, d'un engagement réfléchi, et la validation (Saboya, 2010)2 ${ }^{2}$.

Avant toute résolution, avoir du contrôle peut se traduire par une anticipation qui peut prendre différentes formes : une estimation de l'ordre de grandeur de la réponse ou une analyse préalable des propriétés que devra posséder le résultat (par exemple, repérer qu'il doit être un multiple de trois). L'anticipation peut être associée à la vérification, au retour sur la solution trouvée, mais également au retour sur la méthode qui a permis d'arriver à ce résultat et donc sur les critères de choix de cette méthode (Coppé, 1993). Pour Richard (1995), cette vérification a lieu après l'exécution, et peut conduire à la découverte d'incohérences entre la solution trouvée et d'autres informations données dans le problème, ou encore à un sentiment pour le chercheur de solution d'avoir épuisé l'espace de recherche et d'arriver ainsi à une impasse. Autrement dit, la vérification peut mener à une remise en cause de la représentation construite, à l'abandon de celle-ci et à la construction d'une autre représentation. Le contrôle se traduit également par une vigilance face aux erreurs. La perception des erreurs repose sur la manifestation d'un doute face à la véracité d'un résultat et passe par la question « est-ce que la réponse a un sens? »(Cipra, 1985).

Pour sa part, le contrôle sémantique renvoie à une interprétation en contexte, à une compréhension du sens derrière les concepts mathématiques, par une prise en compte des grandeurs en jeu, de leurs relations et des possibles contraintes (Saboya et al., 2015). De plus, Kargiotakis (1996) relève l'importance d'exercer un engagement réfléchi qui se manifeste par une prise de distance, un arrêt devant le problème avant, en cours et/ou à la fin de la résolution. Un engagement réfléchi peut également survenir après un blocage (Saboya et al., 2015). Ce contrôle est lié à une réflexion sur l'action, à la reconnaissance de conventions, à l'interprétation d'écritures, à l'élève faisant preuve d'un jugement

2 Le concept de contrôle puise à cinq perspectives : la sociologie, l'éducation mathématique, la psychologie cognitive, l'invention en mathématiques et la didactique des mathématiques. 
critique. Finalement, la validation se distingue de la vérification par une appréciation de la pertinence et de l'efficacité de la démarche adoptée, par une justification des différentes étapes de résolution. La validation s'appuie sur les fondements des concepts mathématiques auxquels on réfère pour justifier sa démarche (Lee et Wheeler, 1989 ; Perkins et Simmons, 1988). Pour Balacheff (1987), le processus de validation est fondé sur une analyse du pour et du contre, ce processus étant fortement lié à celui de la preuve (Jeannotte, 2015). Saboya et al. (2015) et Morand (2020) soulignent que le contrôle n'est pas statique ; souvent imbriquées, ses composantes prennent appui les unes sur les autres.

Certains travaux (Bolduc, 2020 ; Saboya, 2010 ; Saboya et Tremblay, 2017) se sont intéressés à cerner les interventions visant à développer un contrôle chez les élèves. Il s'agit par exemple de mettre en place une phase de validation, en interrogeant les élèves sur le sens des concepts à travers un questionnement reposant sur le «pourquoi » et sur le « comment», ou sur un choix efficace de matériel de manipulation, ou encore en encourageant une diversité de stratégies de résolution. Le présent article apporte un éclairage au concept de contrôle, un contrôle interdisciplinaire à travers la proposition d'exemples d'interventions menées en français et réinvesties dans la résolution de problèmes en mathématiques.

\section{Éléments méthodologiques}

Deux recherches collaboratives ont été menées avec des intervenants du primaire à des niveaux différents. Le modèle de recherche collaborative adopté (Bednarz, 2013 ; Desgagné, 1998 ; Desgagné et al., 2001) a pour pivot central une activité réflexive aménagée entre des chercheurs et des intervenants du milieu scolaire. Elle vise une médiation entre le monde de la recherche et celui de la pratique professionnelle : ces deux communautés vont s'unir autour d'un objet de préoccupation commun dans une démarche de coconstruction. Sont alors mises à contribution des manières de faire et de penser propres à chaque communauté. Le chercheur collaboratif est habité par le critère de double vraisemblance, il doit à la fois considérer les préoccupations, les contraintes et les intérêts des enseignants, mais aussi ceux de la recherche. L'objet d'étude des deux recherches collaboratives est lié à la coconstruction d'interventions dans la résolution de 
problèmes en mathématiques visant le développement d'un contrôle chez les élèves du primaire.

Un des deux projets implique deux enseignantes préoccupées par les difficultés de leurs élèves en adaptation scolaire. Elles interviennent en deuxième et troisième année du primaire et accueillent dans leurs classes une orthophoniste ${ }^{3}$. Ces enseignantes ont plus de dix années d'expérience en adaptation scolaire. Cette recherche s'est déroulée sur une période de 4 ans, de 2013 à 2018 avec une interruption en 2015-2016. Ainsi, nous avions comme intention un travail conjoint sur la résolution de problèmes mathématiques de structure additive (Vergnaud, 1982), afin d'implanter une approche par résolution de problèmes teintée par la préoccupation de développer un contrôle chez les élèves. Les problèmes de structure additive, dont nous allons discuter ici, sont les problèmes de transformation et les problèmes de réunion ${ }^{4}$. La deuxième recherche implique une vingtaine d'intervenants, d'enseignants et de conseillers pédagogiques qui interviennent au troisième cycle du primaire dans des milieux défavorisés. Ceux-ci ont des niveaux d'expérience divers, allant d'intervenants nouvellement engagés à chevronnés, certains comptant plus de 20 ans de métier. Cette recherche s'est déroulée sur deux ans (20172019). La préoccupation commune tourne autour des interventions à mettre en place pour favoriser l'engagement et la réussite des élèves face à la résolution de situationsproblèmes et d'application ${ }^{5}$, tout en assurant le développement d'un contrôle. Trois à quatre rencontres réflexives, d'une journée chacune, ont eu lieu par année pour chacun des deux projets.

Les rencontres réflexives ont été alimentées par des éléments théoriques et des données empiriques sur la notion de contrôle, sur la résolution de problèmes et sur des récits de pratique. Il s'agissait d'identifier des difficultés de mobilisation d'un contrôle chez les élèves et de réfléchir ensemble aux interventions déjà menées ou à mener, à court et à plus long termes, pouvant favoriser le développement d'un contrôle. Entre ces rencontres, les participants étaient encouragés à expérimenter dans leur classe. Des

3 Les orthophonistes s'occupent du dépistage, du diagnostic, du traitement et de la prévention des troubles de la parole, de la voix, du langage et des fonctions de communication.

4 Les problèmes de transformation sont ceux où l'on expose un état initial sur lequel une action (transformation d'ajout ou de retrait) est annoncée pour donner lieu à un nouvel état. Ils se distinguent des problèmes de réunion dans lesquels il y a une recherche d'un sous-ensemble (complément, partie) ou de l'ensemble (le tout).

5 Les caractéristiques de ces situations seront explicitées dans la section traitant de la première intervention. 
enregistrements audio-vidéo ont capté les rencontres réflexives et les séances en classe. Les chercheuses s'appuyaient sur les éléments ressortis lors des rencontres réflexives et sur les expérimentations en classe pour animer la rencontre réflexive qui suivait. À travers les échanges, les enseignants en sont venus à proposer des interventions qu'ils menaient en français et qui pourraient être réinvesties en mathématiques, certaines de ces interventions étant déjà mises en place dans leur pratique. Pour mieux comprendre les interventions en français rapportées par les enseignants, les chercheuses ont procédé à la lecture de documents pédagogiques sur lesquels s'appuyaient les enseignants (comme Bédard et Montpetit [2002] et Dugas [2006]) et à la lecture de différents articles scientifiques que nous citons dans cet article. Les interprétations des chercheuses sur ces lectures ont été soumises aux participants, ce qui a permis d'expliciter leur point de vue et de mieux comprendre leur manière de réinvestir ou de penser le réinvestissement de ce qui est fait en français dans le cours de mathématiques, et d'ainsi contribuer à la coconstruction. Les enseignants ont également rapporté des propos tirés de formations suivies avec des conseillers pédagogiques en français. Le processus d'analyse des données repose sur l'identification d'épisodes de discussion, dans les rencontres réflexives et lors des séances avec les élèves, autour du réinvestissement d'interventions en français dans le cours de mathématiques sous l'angle du contrôle.

\section{Trois interventions menées en français et réinvesties dans la résolution d'histoires mathématiques}

Au lieu de problèmes, nous faisons le choix de parler d'histoires mathématiques puisque, comme le précisent les enseignantes en adaptation scolaire, le mot problème a une connotation négative, alors qu'avec le mot histoire, on se rapproche de ce qui est fait en français. Nous rapportons dans ce qui suit trois réinvestissements du travail mené en français dans la résolution d'histoires mathématiques. Pour chacune des interventions, nous explicitons en premier ce qui est fait en français pour ensuite établir le lien avec ce qui a été coconstruit en mathématiques. La première intervention provient du projet mené avec les enseignants du troisième cycle du primaire, la deuxième est issue du projet avec les enseignantes en adaptation scolaire, et la troisième a été discutée dans les deux projets. 


\section{Intervention $1:$ le prédire en français, réinvesti en mathématiques, devient faire parler l'image}

En français, l'emploi de la stratégie prédire se fait avant d'entamer la lecture d'un texte et elle permet aux élèves de se faire une idée de ce qui y sera traité. À travers divers indices, comme le titre, les tableaux, les cartes, les illustrations, les intertitres et des mots qui sont parfois mis en évidence dans le texte, les élèves peuvent formuler des hypothèses sur le sujet du texte, sur les informations qui seront données en rapport au sujet et sur la façon dont ces informations seront présentées (Godbout et al., 2016). Ainsi, selon ces chercheuses, cette stratégie permet aux élèves d'être plus attentifs et prêts à recevoir des informations ; ils se préparent à la lecture en activant leurs connaissances antérieures liées au sujet du texte et à sa structure ${ }^{6}$. De plus, les enseignants du projet collaboratif soulignent que tout le long de sa lecture, l'élève peut réajuster ses hypothèses. L'activité de contrôle se traduit ici par une anticipation qui porte sur le sujet de l'histoire, sur la nature des informations et sur la structure du texte. Cette anticipation sera par la suite vérifiée, l'élève étant amené à confirmer ou à infirmer son anticipation initiale après la lecture du texte. Il est ainsi capable de percevoir de possibles erreurs dans ses hypothèses et de réajuster son anticipation en conséquence.

Dans la recherche collaborative impliquant des intervenants au troisième cycle du primaire, la stratégie prédire a été réinvestie en mathématiques lors de la résolution de situations bâties sur le modèle des situations proposées par le ministère de l'Education du Québec (MEQ, 2006) et liées aux deux compétences « Résoudre une situation-problème mathématique $^{7}$ » et « Raisonner à l'aide de concepts et de processus mathématiques ${ }^{8} »$. Le premier type de situations met en jeu plusieurs concepts, plusieurs données, plusieurs contraintes, divers registres de représentations et un long énoncé (Caron et René de

6 Nous reviendrons sur la « structure » des textes en français dans la section traitant de la deuxième intervention.

7 Le programme de formation de l'école québécoise associe cinq composantes pour ces situations. L'élève est amené à 1) décoder les éléments de la situation-problème, 2) modéliser la situation-problème, 3) appliquer différentes stratégies en vue d'élaborer une solution, 4) valider la solution et 5) partager l'information relative à la solution (MEQ, 2006, p. 127).

8 Quatre composantes sont associées pour ces situations. L'élève est amené à : 1) cerner les éléments de la situation mathématique, 2) mobiliser les concepts et processus mathématiques appropriés à la situation, 3) appliquer des processus mathématiques appropriés à la situation et 4) justifier des actions ou des énoncés en faisant appel à des concepts et à des processus mathématiques (MEQ, 2006, p. 130). 
Cotret, 2007 ; Lajoie et Bednarz, 2016). Les enseignants soulignent que toutes ces informations sont souvent « indigestes » pour les élèves et provoquent de l'anxiété chez certains d'entre eux. Ces situations suivent un même modèle : sur la première page se trouvent le titre et une image, les pages suivantes donnent l'énoncé en mots, parfois accompagné de graphiques ou de tableaux, ainsi que des encadrés qui visent à aider l'élève dans sa résolution. Le titre permet de situer le sujet abordé par l'histoire mathématique, mais c'est souvent l'image qui est digne d'intérêt. Elle n'est pas banale et vise souvent à illustrer de façon fidèle l'histoire.

Ainsi, avant de lire l'énoncé de l'histoire, on peut amener les élèves à faire parler l'image à travers différentes questions : « D'après vous, que nous dit l'image ? », « Que doit-on faire? », « Que cherche-t-on ? ». Avant la lecture de l'énoncé, les élèves sont ainsi amenés à se faire une idée de l'histoire mathématique et parfois même des concepts mathématiques en jeu. De plus, les élèves sont invités à feuilleter le cahier présentant la situation. Ils peuvent aussi survoler les tableaux qu'ils devront remplir pour avancer dans la résolution. Ce procédé encourage la mise en place d'un engagement réfléchi et d'une anticipation. L'anticipation prend ici différentes formes : une anticipation de l'histoire et de la question à laquelle il faudra répondre, mais également autour des concepts mathématiques suggérés par l'image ; et une anticipation qui porte sur le nombre et la nature des tâches à réaliser pour la résolution. Ainsi, avant de lire l'énoncé, les élèves ont une idée de ce qui sera traité dans l'histoire. Ils peuvent alors vérifier si leur anticipation s'est révélée juste et se réajuster si ce n'est pas le cas. Après cette anticipation, les élèves peuvent s'attarder sur les nombres en jeu et déchiffrer les différentes représentations, comme les tableaux ou les diagrammes présentés dans l'énoncé. Ainsi, l'intervention nommée faire parler l'image amène les élèves à ne pas se focaliser sur les nombres en jeu, mais plutôt à développer une idée générale de l'histoire et de sa résolution. On assiste à une décentration des données numériques. Les élèves prennent de l'assurance grâce à l'anticipation qu'ils ont faite et, lors de la lecture de l'histoire, ils peuvent exercer un contrôle sémantique qui se traduit par le repérage des différentes données, de la relation entre ces données, des contraintes et du but à atteindre.

Ainsi, prédire en français et faire parler l'image en mathématiques sont des stratégies qui se situent en amont de la lecture. En mathématiques, les indices pour prédire sont le titre, l'image associée et le survol des tableaux à remplir. Dans ces deux disciplines, les élèves sont amenés à anticiper l'histoire et à décoder les diverses 
informations. Dans le cas des mathématiques, faire parler l'image porte sur les concepts mathématiques en jeu, mais également sur la résolution en anticipant les diverses étapes à accomplir pour résoudre l'histoire. Dans les deux disciplines, une vérification s'effectue à la suite de l'anticipation lors de la lecture de l'histoire : l'élève peut alors percevoir des erreurs qui vont amener à des réajustements, donc à revoir son anticipation. Les élèves exercent ainsi un engagement réfléchi et un contrôle sémantique sur l'histoire mathématique. Toutefois, les enseignants font le constat que toutes les images ne sont pas parlantes. Dans le projet, des images parlantes ont été construites par les enseignants et il a également été proposé à des élèves d'en élaborer.

\section{Intervention 2 : le récit en trois temps et le texte informatif en français réinvestis dans les problèmes de structure additive en mathématiques}

Cette intervention a eu lieu dans le cadre du projet mené avec les deux enseignantes de deuxième et troisième années du primaire en adaptation scolaire. Celles-ci rapportent des difficultés ressenties par les élèves dans la résolution d'histoires mathématiques de structure additive. Elles affirment que les élèves prennent souvent les nombres qu'ils trouvent dans l'histoire et les ajoutent sans se demander si la réponse a du sens. Les élèves ont alors des difficultés à percevoir la structure de l'histoire. Ces intervenantes ont alors pensé à ce qu'elles font en français : elles amènent les élèves à reconnaître la structure du texte. À cet effet, Godbout et al. (2016) soulignent l'importance pour un élève de reconnaître la structure d'un texte afin d'être mieux à même de : « se rappeler des informations lues, d'identifier les extraits importants, de se repérer dans le texte et d'identifier ses pertes de compréhension » (p. 19). Par «structure », Bédard et Montpetit (2002) entendent « l'organisation des informations données dans le texte» (p. 87). En effet, tout texte possède une organisation, une structure propre. Dans l'enseignement du français, plusieurs chercheurs (Dickson et al., 1998 ; Williams, 2003, 2005 ; Williams et al., 2004, 2005, 2007) cités par Godbout et al. (2016) soutiennent que les « connaissances sur l'organisation du texte affectent positivement la compréhension » (p. 19). Bédard et Montpetit (2002) vont dans le même sens. Lors des rencontres réflexives, les enseignantes font part des liens qui existent entre les structures de textes en français du récit en trois temps et du texte informatif, et les histoires de transformation dans le temps et de réunion avec recherche d'un état en mathématiques. 
Réinvestir le récit en trois temps en français dans les histoires de transformation dans le temps en mathématiques. Les enseignantes font référence à Dugas (2006) qui s'est intéressée à la séquence narrative qui repose sur un ensemble de règles appelé la grammaire du récit et qui comprend cinq parties :

1. la situation initiale, qui présente le personnage principal et la situation dans laquelle il se trouve au début de l'histoire (le temps, le personnage, le lieu, l'intention);

2. l'élément déclencheur, qui est l'événement qui bouleverse la situation initiale (le problème) ;

3. le nœud, c'est-à-dire la suite d'actions entreprises par le personnage principal pour résoudre le problème posé par l'élément déclencheur (la solution);

4. le dénouement, qui est l'élément ultime qui mène à une amélioration ou une dégradation de la situation (la fin);

5. la situation finale, qui arrive après le dénouement (partie parfois absente). (Chartrand et al., 1999, cité dans Dugas, 2006, p. 1)

Pour simplifier le vocabulaire de la séquence narrative, Dugas a établi des motsclés pour chacune des parties qui deviennent des marqueurs de cohésion dans le récit. Ces mots-clés sont illustrés par des pictogrammes. Le tableau 1 présente les cinq parties de la séquence narrative avec les composantes du récit, les mots-clés, les pictogrammes et un exemple. Le dénouement et la situation finale (parties 4 et 5) sont représentés par un même pictogramme et mot-clé, et la situation initiale (partie 1) par quatre pictogrammes et mots-clés. 


\section{Tableau 1}

Les cinq parties de la séquence narrative avec les pictogrammes et mots-clés associés

\begin{tabular}{|c|c|c|c|}
\hline $\begin{array}{c}\text { La séquence } \\
\text { narrative (5 } \\
\text { parties) }\end{array}$ & $\begin{array}{l}\text { Composantes du } \\
\text { récit }\end{array}$ & $\begin{array}{c}\text { Pictogrammesav } \\
\text { mots clés }\end{array}$ & Un exemple \\
\hline \multirow{4}{*}{$\begin{array}{l}\text { La situation } \\
\text { initiale }\end{array}$} & Temps & & Unjour, \\
\hline & Personnage & & il y avait quelqu'un, \\
\hline & Lieu & & quelque part \\
\hline & Intention & & $\begin{array}{l}\text { qui voul ait quel que } \\
\text { chose dans son coeur. }\end{array}$ \\
\hline $\begin{array}{l}\text { L'élément } \\
\text { déclencheur }\end{array}$ & Problème & & Mais ahnon! \\
\hline Le noud & Idée & & Idée. \\
\hline $\begin{array}{l}\text { La solution-le } \\
\text { dénouement }\end{array}$ & Finalem ent & Finalement... & $\begin{array}{l}\text { Finalem ent a-t-il eu ce } \\
\text { qu'il voul ait dans son } \\
\text { coeur ou non? Peut-il } \\
\text { continuer ses activités? }\end{array}$ \\
\hline
\end{tabular}

Note. Tableau produit à partir de Dugas (2006, p. 24, 25 et 27).

Pour rendre le récit plus accessible aux jeunes qui vivent des difficultés de toutes sortes, Dugas (2006) propose de regrouper certaines parties du récit en trois temps : le début (partie 1), le milieu (parties 2 et 3) et la fin (parties 4 et 5), comme suit :

\begin{tabular}{c|c|c|c|c|}
\hline \multicolumn{2}{c|}{ DÉBUT } & MILIEU & FIN \\
\hline Personnage & Lieu & Intention & $\begin{array}{c}\text { Évènement } \\
\text { (Que se passe-t-11?) }\end{array}$ & Situation finale \\
\hline
\end{tabular}


Après avoir lu le texte, l'élève est amené à reconnaître sa structure. Pour cela, les enseignantes pigent dans un sac l'un des sept pictogrammes - précisons que le temps auquel appartient le pictogramme est indiqué au verso. Elles questionnent ensuite les élèves sur les parties du texte qui traitent du temps pigé. Cette façon de procéder favorise un engagement réfléchi de la part des élèves, un temps d'arrêt devant le texte à lire qui facilite une reconnaissance des différents temps du récit. Est alors mobilisé un contrôle sémantique qui permet une identification de certaines parties du texte aux différents temps. Les enseignantes s'assurent ainsi de la compréhension de l'histoire par les élèves.

En mathématiques, les enseignantes de deuxième et troisième année et l'orthophoniste font un lien entre le récit en trois temps et les problèmes de transformation dans le temps. En effet, ces derniers problèmes présentent trois temps : le début, le milieu et la fin. Comme en français, le milieu est associé à un « tout à coup » qui marque une surprise, un changement dans la situation initiale. Prenons, par exemple, l'histoire de transformation dans le temps avec recherche de l'état initial proposée par l'enseignante de deuxième année au début de la mise en place de son intervention en mathématiques :

Cette année, à l'école, il y a un concours de fléchettes. L'élève gagnant est celui qui a accumulé le plus grand nombre de points. Il dit : «Au début, je n’avais pas beaucoup de points. Ensuite, je me suis amélioré et j'ai fait 42 points ! J'ai gagné parce que j'ai terminé le concours avec 68 points ! Combien de points avais-je au début du concours?»

L'enseignante évoque avec les élèves ce qui est fait en français pour les amener à exercer un meilleur contrôle lors de la résolution de problèmes en mathématiques. Elle écrit les trois temps au tableau : début, milieu et finalement. Comme en français, elle les amène à reconnaître les parties de l'histoire qui se situent au début, au milieu et à la fin en utilisant les mêmes pictogrammes. Il s'agit, de plus, en mathématiques, de trouver ce qu'elle nomme le nombre mystère. Voici un extrait de l'échange dans lequel le travail de repérage des différents temps est souvent pris en charge par l'enseignante nommée Marie. Nous avons ajouté entre crochets ce qu'elle écrit et pointe au fur et à mesure au tableau (voir figure 2$)^{9}$.

9 Le lecteur peut se référer à Fillion (2020) et Fillion et al. (2019) pour une analyse plus complète de cet extrait. 
Marie : Au début, il y avait des points [elle pointe avec sa main sur le tableau le début en haussant les épaules]. MAIS, est-ce qu'on savait combien ? [Elle met l'accent sur le mot « mais » en levant le ton légèrement et écrit un point d'interrogation].

Élèves : Non.

Marie : Après, on savait qu'il en a fait 42. Il avait gagné, car il avait obtenu 68 points [déplace sa main sur chacun des mots « milieu » et « finalement » et écrit 42 et 68$]$.

Marie : Notre question c'était combien il a fait de points [avec sa main, elle pointe le « début »].

Élèves : Au début.

Marie : Au DÉBUT [Elle met l'accent sur le mot « début » en levant le ton légèrement].

\section{Figure 2}

Mathématisation par l'enseignante de deuxième année de l'histoire des fléchettes

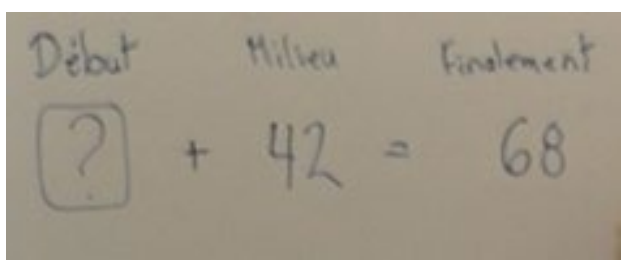

Ce travail de mathématisation de l'histoire repose sur la mobilisation d'un engagement réfléchi qui se traduit par un arrêt devant l'énoncé pour détecter d'abord la structure du texte, puis les trois temps de l'histoire. Par la suite, le recours à un contrôle sémantique appuie la mathématisation de l'histoire en une égalité.

\section{Réinvestir le texte informatif en français pour travailler des histoires de réunion} avec recherche d'un état en mathématiques. En troisième année du primaire en français, les élèves sont initiés aux textes informatifs. Dans ce type de texte, le sujet est porté par des idées principales. L'enseignante et l'orthophoniste utilisent la schématisation suivante avec les élèves : 


\section{Figure 3}

Schématisation utilisée avec les élèves de troisième année primaire en adaptation scolaire pour travailler le texte informatif

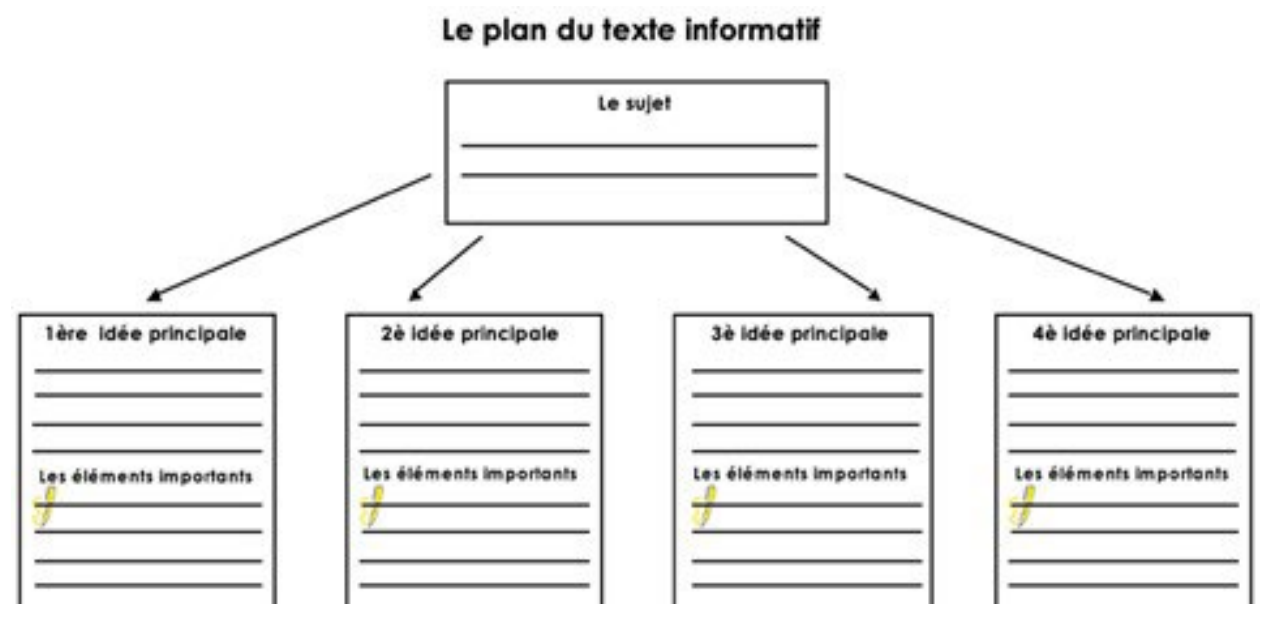

En mathématiques, les éléments du texte informatif sont repris pour les histoires de réunion pour lesquelles il y a des «parties » et un « tout» ou un « total». Les parties rassemblées vont permettre de retrouver le «tout ». Prenons l'histoire mathématique suivante proposée par l'enseignante de troisième année : « À l'école de Julien, il y a 623 élèves. Son enseignant lui dit qu'il y a 297 garçons. Combien il y a de filles à l'école de Julien ?»

Le « tout » est constitué du nombre total d'élèves, ici les 623 élèves, et il y a 2 « parties », le nombre de garçons qui est connu et le nombre de filles qui est recherché, c'est le nombre mystère. Lors de la résolution de cette histoire mathématique, l'enseignante questionne les élèves sur le sujet traité et également sur les idées principales, faisant ainsi un lien avec la structure du texte informatif en français. Ce lien est explicité aux élèves, le sujet est le « tout» ou le « total », et les idées principales constituent les différentes parties du sujet, du « tout» ou du « total» (voir tableau 2). Les élèves statuent facilement que le nombre de filles plus le nombre de garçons seront égal au nombre d'élèves. 


\section{Tableau 2}

Parallèle entre les textes informatifs en français et les histoires de réunion en mathématiques

\begin{tabular}{c|c}
\hline En français & En mathématiques \\
\hline SUJET & $\begin{array}{c}\text { TOUT } \\
\text { Le nombre total d'élèves } \\
\text { Partie 2 } \\
\text { Partie 1 }\end{array}$ \\
Idéeprincipale 1 Idée principale 2 & $\begin{array}{c}\text { Le nombre de gar çons } \\
\text { Lombre de filles + nom bre de garçons = nom bre d'élèves }\end{array}$ \\
\hline
\end{tabular}

L'enseignante précise que le lien entre les textes informatifs et les histoires de réunion en mathématiques devient intéressant avec des histoires qui demandent une connaissance d'un vocabulaire spécialisé. Elle donne l'exemple de l'histoire suivante :

À la pépinière près de chez moi, il y a 581 arbres à vendre. Le pépiniériste m'a dit qu'il y a 208 conifères parmi ces arbres. Ensuite, il m'a demandé de calculer combien il y a de feuillus. Aide-moi à calculer le nombre de feuillus.

Après avoir expliqué ce qu'est une pépinière, des conifères et des feuillus, elle amène les élèves à schématiser que le nombre total d'arbres constitue le « tout », ou le sujet, et que les deux parties sont le nombre de conifères et le nombre de feuillus. Les élèves repèrent ainsi la structure de l'histoire et voient que le nombre de conifères plus le nombre de feuillus seront égal au nombre d'arbres.

Comme pour le récit en trois temps, dans le texte informatif, les élèves sont amenés à identifier la structure du texte, ce qui requiert un engagement réfléchi qui repose sur la reconnaissance d'indices qui vont leur permettre de se situer dans les éléments du texte. Bédard et Montpetit (2002) soulignent que la représentation graphique, ou schématisation, d'un texte informatif aide l'élève à réfléchir sur sa lecture et à établir des liens entre les divers éléments du texte. On peut dire que les élèves exercent dans ce cas un engagement réfléchi par une prise de distance pour repérer la structure. C'est ce même type de contrôle qui est requis des élèves quand ils sont face à des histoires 
mathématiques de structure additive. Comme le précisent souvent les enseignantes aux élèves, « il y a comme un petit cinq minutes que vous devez prendre, puis là vous vous dites : “oh, je fais quoi ?” " (enseignante de 3e année), « Il faut que vous réfléchissiez, puis des fois vous allez voir que, "ah non, finalement ça ne marche pas“" (enseignante de 2e année).

\section{Intervention 3 : la justification en français et en mathématiques suscitée par le « parce que »}

En français, les enseignantes en adaptation scolaire précisent qu'elles apprennent aux élèves à réagir et à exprimer leur jugement à la suite de la lecture d'un texte. Elles utilisent des questions de réaction en lecture et de jugement critique. Elles demandent souvent aux élèves « Pourquoi tu aimes cette histoire-là ? Parce que... ». Elles soulignent que les élèves disent souvent : « parce que je l'aime » ou « parce qu'elle est belle ». Les enseignantes réagissent alors en soulignant aux élèves que l'on peut dire ceci de n'importe quelle histoire ; elles souhaitent alors amener les élèves à développer le « parce que » en leur demandant de donner des arguments en lien avec des éléments de l'histoire. En mathématiques, dans les situations de validation associées à la seconde compétence ou lors de l'explication de la résolution, elles demandent aux élèves de justifier leur réponse à l'aide de concepts, de propriétés ou de relations mathématiques. Comme le précise Jeannotte (2015), la justification repose sur « la recherche de données, de permis d'inférer, de recours à des fondements mathématiques afin de modifier la valeur épistémique d'un énoncé » (p. 112). L'enseignante de troisième année rapporte un exemple tiré de sa pratique :

Quand on fait de la mesure, on disait aux élèves : «Un décimètre, est-ce que c'est plus grand ou plus petit que treize centimètres ? ». Là on ajoute « parce que », ça oblige les élèves soit à aller chercher le mètre ou une mesure, de montrer ou de dire : « parce que un décimètre c'est dix centimètres, et dix centimètres, c'est plus petit que treize centimètres $»$.

Ainsi, que ce soit en français ou en mathématiques, l'élève est invité à justifier sa réponse. En didactique du français, Forget (2012) précise que « justifier consiste globalement à fournir les raisons ou les motivations visant à rendre un acte, une 
croyance, une position ou une réponse recevable aux yeux de son interlocuteur ou de son destinataire » (p. 238). Pour cela, l'élève

présente d'abord ce qui fait l'objet de la justification (sa réponse, sa position ou sa proposition) puis [il] introduit, par un connecteur de type « parce que », les éléments ou les raisons qui appuient cette position, cette proposition ou cette réponse avant de conclure. (Adam, 1992, Duval, 1992, Zakhartchouk, 2001, cités dans Forget, 2014, p. 40-41)

Au primaire, lors de la lecture de textes, la justification de l'élève s'appuie souvent sur des informations qui sont déjà présentes dans le texte. Lors de discussions, l'élève sera invité à argumenter son point de vue en s'appuyant sur son bagage culturel. En mathématiques, la justification peut de plus s'appuyer sur ses connaissances mathématiques ; comme en français, l'enseignant peut encourager la justification lors d'échanges entre pairs ou en grand groupe. C'est ce que nous illustrerons maintenant.

Le recours au « parce que » a été également cité dans la recherche collaborative impliquant des participants intervenant au troisième cycle du primaire. Comme en français, les enseignants ont recours à la question « Pourquoi ? » à différents moments de la résolution d'une histoire mathématique. L'exemple que nous avons choisi de présenter ici illustre la richesse du « parce que » lors d'une discussion entre deux élèves placés en dyade autour de la résolution de l'histoire suivante :

\section{La guignolée}

Les écoles de Lachine ont ramassé des denrées pour la guignolée. À partir de toutes les denrées recueillies, des paniers contenant 10 produits seront livrés à des familles dans le besoin. Sachant que l'école Philippe-Morin a réussi à rassembler 327 produits, combien de paniers seront livrés aux familles?

Après avoir résolu individuellement l'histoire mathématique, l'enseignante demande aux élèves de justifier leur résolution à l'un de leurs camarades. Dans une des équipes, la considération du reste n'est pas interprétée de la même façon par les deux enfants. L'une des élèves (identifiée par E1) donnera comme réponse 32 paniers, alors que l'autre (identifiée par E2) optera pour 33 paniers. S'enclenche alors une discussion autour du «c'est 32 ou 33 paniers parce que » : 
E1 : On a sept produits, mais il en manque trois pour faire un paquet de dix. Ils sont où les trois? Est-ce que tu fais abracadabra et ils arrivent?

E2 : Oui, mais si... 32,7 on l'arrondit aux unités près, ça donne 33. Parce que sept c'est plus grand que cinq, et c'est plus proche d'un entier.

E1 : Oui, mais ils sont où les trois qu'il faut que tu aies pour...

Les arguments évoqués par les deux élèves ne sont pas de même nature. E1 donne du sens au reste en contexte, influencée par la vie réelle, alors que E2 décontextualise et donne un argument de type mathématique sur l'arrondi des nombres. Pour convaincre E1, E2 reprend un argument en contexte, l'argument mathématique est toutefois bien présent :

E2 : C'est des produits, mais là on ne dit pas... Il a dit dix produits pour des familles, alors peut-être que dix produits on le donne à une famille. Mais là le sept il aura moins, mais ce sera quand même assez. Parce que sept il en reste juste trois pour que ça fasse dix, un entier. Si c'est quatre on va le mettre ailleurs parce que c'est plus petit que cinq.

E1 : Oui, mais sinon,... C'est la guignolée, donc les pauvres risquent d'en avoir moins. C'est des paquets de dix.

N'ayant pas réussi à convaincre son interlocutrice, E2 change alors de contexte, on peut faire l'hypothèse qu'elle souhaite laisser de côté la question de la pauvreté sur laquelle elle n'a pas d'arguments assez crédibles et valables pour faire changer d'avis sa partenaire. Son argument portera fruit, E1 avancera que la considération sur le reste diffère selon le contexte. Les élèves ne statuent toutefois pas sur le contexte de la guignolée :

E2 : Si par exemple c'est un album et on met des timbres. S'il y a en tout cinq pages, à chaque page il faut avoir,... Il y a en tout six pages et chaque page doit avoir cinq timbres, il va en rester, par exemple. Et le nombre c'est 26, il va en rester un, alors on va le mettre dans l'autre page et pas l'insérer ou le mettre ailleurs.

E1 : Mais est-ce qu'on est dans le contexte qu'on arrondit pour faire ça, ou est-ce qu'on est juste,... Parce que dans quelques contextes, comme avec de l'argent, 32 et 7 dixièmes, on va donner 33 dollars. Mais dans ce contexte, est-ce qu'on est vraiment avec de l'argent ou avec un autre contexte ? 
Nous pouvons remarquer que la demande de justification initiale de l'enseignante se mue en une argumentation entre les deux élèves. Forget (2014) souligne que les finalités de la justification et de l'argumentation sont différentes. En effet, la justification ne cherche pas à convaincre le destinataire d'adopter une thèse sur un sujet controversé où s'affrontent deux thèses opposées. Forget (2014) précise, en s'appuyant sur Grize (1996), que l'argumentation se construit dans « une logique et avec la langue naturelle : elle recourt à des arguments crédibles pour les parties puisque c'est la valeur de vraisemblance qui prévaut » (p. 44). L'argumentation cherche à persuader, à convaincre (Thyrion, 1997); la prise en compte de son destinataire sert à anticiper les arguments de ce dernier afin de s'en servir ou de mieux les réfuter. On s'aventure sur le terrain de l'adversaire tout en gardant une position offensive (Forget, 2014). En didactique des mathématiques, Cabassut (2005) relie l'argumentation à la validation de la plausibilité, la probabilité de la vérité d'une proposition. Ainsi, la vérité de la conclusion n'est pas nécessaire, mais plus ou moins plausible. On assiste bien dans la discussion entre les deux élèves à une argumentation.

Le contrôle exercé en français et en mathématiques se mobilise autour de la justification et de l'argumentation, qui sont reliées à la validation (Cabassut, 2005). Hubert (2015) précise que ces interventions autour du « parce que » sont importantes, elles permettent de développer une attitude critique chez les élèves que l'on peut relier au contrôle : «Il s'agit d'amener les élèves à mettre en doute leur position, à envisager d'autres perspectives (celles de leur destinataire principalement), à s'assurer de la crédibilité des faits, des preuves et des informations qu'ils convoquent » (s. p.).

\section{Discussion}

Cet article s'intéresse aux interventions d'enseignants du primaire qui établissent des liens entre des processus d'apprentissage développés et mobilisés dans des activités d'enseignement-apprentissage vécues en français et en mathématiques. Il se distingue des recherches dans lesquelles on s'attarde aux élèves à travers leurs capacités de lecture en français et en résolution de problèmes (Cummins et al., 1988 ; Vilenius-Tuohimaa et al., 2008) ou même leur habileté à produire des inférences (Luquette, 2017 ; Voyer et al., 2012). C'est l'enseignant qui est au centre de cette étude à travers un réinvestissement 
dans la résolution de problèmes en mathématiques des stratégies de prédire, du récit en trois temps, du texte informatif et du parce que utilisées en français. Il en résulte trois interventions qui visent à développer une activité de contrôle transversale aux deux disciplines. Ce contrôle s'exerce à travers le recours à un engagement réfléchi, un contrôle sémantique, une anticipation et une validation, cette dernière prenant la forme d'une justification et d'une argumentation. Plus particulièrement, l'intervention prédire en français a été nommée faire parler l'image en mathématiques. Toutefois, soulignons que dans cette discipline, on peut remonter au début du 20e siècle pour retrouver le décodage d'images. En effet, dans un livre de première année du primaire au Québec datant de 1922, dont les auteurs sont Magnan et Ahern, différentes images présentent des scènes de la vie réelle. Cependant, les images proposées ont une fonction différente de celle que nous avons dégagée dans cet article. Il s'agissait alors essentiellement pour les enfants de dénombrer des objets et de procéder aux quatre opérations de base ; aucune histoire mathématique écrite ne suivait la présentation de l'image.

Les interventions présentées dans cet article permettent de constater que les textes travaillés en français, dans ce cas-ci les textes narratifs et informatifs, peuvent être reliés à certains types de problèmes additifs en mathématiques, ceux de transformation dans le temps et de réunion. Cet intérêt à propos des structures des textes a été étudié par Voyer et al. (2012), qui rapportent qu'en quatrième année du primaire, le rendement en résolution de problèmes arithmétiques est davantage lié à la compréhension de textes informatifs que de textes narratifs, mais que cet effet de la structure du texte semble s'atténuer au cours de la scolarité en ce qui concerne le rendement en mathématiques en sixième année du primaire. Dans la poursuite de cette recherche, il serait intéressant d'étudier l'impact de ces interventions sur la résolution de problèmes menée par les élèves.

Ainsi, cette étude s'inscrit dans les recherches portant sur l'interdisciplinarité qui vise la création de liens entre les disciplines en assurant un apprentissage plus réel, concret et global (Lowe, 2002). Lowe ajoute que l'interdisciplinarité scolaire doit favoriser une pédagogie du vécu et de la découverte, éléments fondamentaux de la pédagogie « actualisante » qui se veut intégrative. Lenoir et Sauvé (1998) précisent qu'une approche interdisciplinaire consiste en la

mise en relation de deux ou plusieurs disciplines scolaires [...] qui conduit à l'établissement de liens de complémentarité ou de coopération, d'interprétation 
ou d'actions réciproques entre elles sous divers aspects (finalités, objets d'étude, concepts et notions, démarches d'apprentissage et habiletés techniques, etc.) en vue de favoriser l'intégration des processus d'apprentissage et des savoirs chez les élèves. (p. 12)

Par la mise en place d'une approche par résolution de problèmes dans les classes de mathématiques, les enseignants de nos deux projets de recherche collaborative reconnaissent, d'une part, que plusieurs de leurs élèves adoptent une attitude d'ouverture aux idées différentes et un désir de comprendre qui se transpose dans les différentes disciplines et, d'autre part, les enseignants admettent sans hésiter prendre la posture d'enseignant « questionneur », peu importe la discipline enseignée. Nous souhaitons que la mise en place des interventions présentées ici participe à une intégration des apprentissages, permettant ainsi à l'élève de donner une signification à son apprentissage et de lui trouver un sens (Lenoir et Sauvé, 1998). De plus, ces deux recherches collaboratives ont permis aux chercheuses d'explorer le champ de la didactique du français et de l'interdisciplinarité, ouvrant ainsi la voie à des interventions novatrices porteuses d'un contrôle.

\section{Conclusion}

L'implantation d'une approche par résolution de problèmes soutenue par la recherche de l'expression d'un contrôle peut motiver les enseignants à tisser des liens entre des processus mobilisés en mathématiques et en français. Du côté des recherches en didactique, les interventions dégagées dans le présent article invitent à revisiter l'analyse du façonnement des manières d'agir et de penser des élèves en mathématiques, particulièrement au primaire, pour ainsi rendre compte de ce qui est fait et dit « en dehors » des périodes destinées à l'apprentissage des mathématiques. Ils alimentent la discussion autour des liens entre la résolution de problèmes en mathématiques et les habiletés développées en français sous l'angle des interventions des enseignants, un aspect peu traité dans la littérature. Cette étude ouvre la porte aux interventions dans plusieurs disciplines, pas seulement en français et en mathématiques comme rapporté ici, mais également en sciences et en univers social. L'étude de la validation à travers l'argumentation, les explications, les justifications, la preuve et la démonstration est aussi 
une piste intéressante à suivre pour une approche interdisciplinaire. Le recours au parce que nous semble intéressant à exploiter puisqu'il traduit l'importance de la justification et de l'argumentation en mathématiques et en français, mais quelle forme prend-il dans les autres disciplines ? Ces réflexions pourraient contribuer à la formation des enseignants. 


\section{Références}

Adam, J.-M. (1992). Les textes : types et prototypes. Récit, description, argumentation, explication et dialogue. Nathan.

Balacheff, N. (1987). Processus de preuve et situations de validation. Educational Studies in Mathematics, 18(2), 147-176. https://doi.org/10.1007/BF00314724

Bédard, D. et Montpetit, D. (2002). Stratégies... stratégies... pour une lecture efficace au primaire : de la théorie à la pratique. CEC.

Bednarz, N. (dir.). (2013). Recherche collaborative et pratique enseignante : regarder ensemble autrement. L'Harmattan.

Bolduc, A. (2020). Analyse d'interventions menées par une orthopédagogue du secondaire qui contribuent à l'expression d'un contrôle en mathématiques chez des élèves en difficulté d'apprentissage [Mémoire de maîtrise, Université du Québec à Montréal]. Archipel. http://archipel.uqam.ca/id/eprint/14181

Cabassut, R. (2005). Démonstration, raisonnement et validation dans l'enseignement secondaire des mathématiques en France et en Allemagne (HAL Id : tel00009716) [Thèse de doctorat, Université Paris Diderot-Paris VII]. Archivesouvertes.fr.

Caron, F. et René de Cotret, S. (2007, 6-8 juin). Un regard didactique sur l'évaluation en mathématiques : genèse d'une perspective. Dans P. Marchand (dir.), Actes $d u$ colloque GDM2007. La didactique des mathématiques au Québec : genèse et perspectives (p. 123-134). https://www.academia.edu/21829191/

Chartrand, S.-G, Aubin, D., Blain, R. et Simard, C. (1999). Grammaire pédagogique du français d'aujourd'hui. Graficor.

Cipra, B. (1985). Erreures... et comment les trouver avant le prof ... . InterEditions.

Coppé, S. (1993). Processus de vérification en mathématiques chez les élèves de première scientifique en situation de devoir surveillé [Thèse de doctorat inédite]. Université de Lyon 1, France. 
Cummins, D. D., Kintsch, W., Reusser, K. et Weimer, R. (1988). The role of understanding in solving word problems. Cognitive Psychology, 20(4), 405-438. https://doi.org/10.1016/0010-0285(88)90011-4

Desgagné, S. (1998). La position du chercheur en recherche collaborative : illustration d'une démarche de médiation entre culture universitaire et culture scolaire. Recherches qualitatives, 18, 77-105.

Desgagné, S., Bednarz, N., Couture, C., Poirier, L. et Lebuis, P. (2001). L'approche collaborative de recherche en éducation : un nouveau rapport à établir entre recherche et formation. Revue des sciences de l'éducation, 27(1), 33-64. https:// doi.org/10.7202/000305ar

Devidal, M., Fayol, M. et Barrouillet, P. (1997). Stratégies de lecture et résolution de problèmes arithmétiques. L'année psychologique, 97(1), 9-31. https://doi. org/10.3406/psy.1997.28935

Dickson, S. V., Simmons, D. C. et Kame'enui, E. J. (1998). Text organization: Research bases. Dans D. C. Simmons et E. J. Kame'enui (dir.), What reading research tells us about children with diverse learning needs: Bases and basics (p. 239-278). Erlbaum.

Dufour, S. et Jeannotte, D. (2013). La tâche non routinière sous l'angle du contrôle : un exemple en calcul différentiel. Bulletin $A M Q, 53(4), 29-43$. https://www.amq. math.ca/ancien/bulletins/dec13/05-maitre-Dufour-Jeannotte.pdf

Dugas, B. (2006). Le récit en $3 D$. Chenelière Éducation.

Duval, R. (1991). Interaction des niveaux de représentation dans la compréhension de textes. Annales de Didactique et de Sciences Cognitives, 4, 163-196. https:// mathinfo.unistra.fr/websites/math-info/irem/Publications/Annales_didactique/ vol 04/adsc4_1991-006.pdf

Duval, R. (1992). Argumenter, démontrer, expliquer : continuité ou rupture cognitive ? Petit x, (31), 37-61. https://irem.univ-grenoble-alpes.fr/medias/ fichier/31x5 1570192011747-pdf

Fagnant, A., Dupont, V. et Demonty, I. (2016). Régulation interactive et résolution de tâches complexes en mathématiques. Dans L. Mottier Lopez et W. Tessaro (dir.), Le jugement professionnel au cour de l'évaluation et de 
la régulation des apprentissages (p. 229-251). Peter Lang. https://doi. org/10.3726/978-3-0352-0340-0

Fayol, M. (1992). Comprendre ce qu'on lit : de l'automatisme au contrôle. Dans M. Fayol, J. E. Gombert, P. Lecocq, L. Sprenger-Charolles et D. Zagar (dir.), Psychologie cognitive de la lecture (p. 73-105). PUF.

Fillion, A. (2020). Analyse de l'activité d'enseignement-apprentissage du concept de nombre sous une approche par résolution de problèmes chez une enseignants de 2 e année en adaptation scolaire (publication no 1245862669). [Mémoire de maîtrise, Université du Québec à Rimouski]. WorldCat.

Fillion, A., Tremblay, M. et Saboya, M. (2019, 24-26 mai). La théorie de l'objectivation pour cerner l'enseignement/apprentissage dans une approche par résolution de problèmes mathématiques dans une classe de 2e année en adaptation scolaire. Dans C. Corriveau, V. Martin, M. Thibeault et A. Savard (dir.), Actes du colloque GDM2019. À quoi ressemble aujourd'hui la recherche en didactique des mathématiques au Québec? (p. 109-121). Université Laval. https://www.gdm. quebec/colloque/actes

Forget, M.-H. (2012). Places et rôles de l'oral dans l'activité d'écriture d'une justification : une « ontométhode »? Dans R. Bergeron et G. Plessis-Bélair (dir.), Représentations, analyses et descriptions du français oral, de son utilisation et de son enseignement au primaire, au secondaire et à l'université (p. 237-252). Peisaj.

Forget, M.-H. (2014). Pratiques d'écriture de justifications d'élèves plurilingues du ler cycle du secondaire. Entre savoirs d'expérience et apprentissages en classe de français [Thèse de doctorat, Université Sherbrooke]. Savoirs UdeS. http://hdl. handle.net/11143/5373

Freiman, V. et Savard, A. (2014). Résolution de problèmes en mathématiques. Éducation et francophonie, 42(2), 1-6. https://doi.org/10.7202/1027902ar

Ge, X. et Land, S. M. (2003). Scaffolding students' problem-solving processes in an ill-structured task using question prompts and peer interactions. Educational Technology Research and Development, 51(1), 21-38. https://doi.org/10.1007/ BF02504515 
Giroux, J. (2004). Échanges langagiers et interactions de connaissances dans l'enseignement des mathématiques en classe d'adaptation scolaire. Revue des sciences de l'éducation, 30(2), 303-327. https://doi.org/10.7202/012671ar

Godbout, M.-J., Turcotte, C. et Giguère, M.-H. (2016). 8 stratégies pour comprendre les textes courants : 4e, 5e et 6e année du primaire, guide pédagogique. https:// adel.uqam.ca/wp-content/uploads/2020/12/guide-pedagogique-8-strategies-pourcomprendre-les-textes-courants.pdf

Goulet, M.-P. (2013). L'effet de l'habileté en lecture, selon la structure du texte et le type de question administrée, sur le rendement en résolution de problèmes écrits d'arithmétique [Mémoire de maîtrise, Université du Québec à Rimouski]. Sémaphore. http://semaphore.uqar.ca/id/eprint/957

Goulet, M.-P. et Voyer, D. (2014). La résolution de problèmes écrits d'arithmétique : le rôle déterminant des inférences. Éducation et francophonie, 42(2), 100-119. https://doi.org/10.7202/1027908ar

Grize, J.-B. (1996). Logique naturelle et communications. PUF.

Hubert, B. (2015, 14 avril). Justifier ses propos : entre savoirs d'expérience et nouveaux apprentissages. http://rire.ctreq.qc.ca/2015/04/justification-apprentissage/

Jeannotte, D. (2015, 20-22 mai). Proposition d'un modèle de raisonnement mathématique pour l'apprentissage au primaire et au secondaire dans une perspective commognitive. Dans A. Adihou, L. Bacon, D. Benoit et C. Lajoie (dir.), Actes $d u$ colloque GDM2015. Regard sur le travail de l'enseignant de mathématiques (p. 109-118). https://www.academia.edu/39757588/

Kargiotakis, G. (1996). Contribution à l'étude de processus de contrôle en environnement informatique : le cas des associations droites-équations [Thèse de doctorat inédite]. Université Paris VII - Denis Diderot, France.

Kemmerle, M. (2013). Promoting student questions in mathematics classrooms. Dans M. Martinez et A. Castro Superfine (dir.), Proceedings of the 35th annual meeting of the North American Chapter of the International Group for the Psychology of Mathematics Education (p. 1004-1011). University of Illinois at Chicago. https:// files.eric.ed.gov/fulltext/ED584475.pdf 
Lajoie, C. et Bednarz, N. (2012). Évolution de la résolution de problèmes en enseignement des mathématiques au Québec : un parcours sur cent ans des programmes et documents pédagogiques. Revue canadienne en enseignement des sciences, de la technologie et des mathématiques, 12(2), 178-213. http://dx.doi. org/10.1080/14926156.2012.679992

Lajoie, C. et Bednarz, N. (2016). La notion de situation-problème en mathématiques au début du XXIe siècle au Québec : rupture ou continuité ? Revue canadienne d'enseignement des sciences, des mathématiques et des technologies, 16(1), 1-27. http://dx.doi.org/10.1080/14926156.2014.993443

Lee, L. et Wheeler, D. (1989). The arithmetic connection. Educational Studies in Mathematic, 20(1), 41-54. https://doi.org/10.1007/BF00356040

Lenoir, Y. et Sauvé, L. (1998). Introduction. L'interdisciplinarité et la formation à l'enseignement primaire et secondaire : quelle interdisciplinarité pour quelle formation ? Revue des sciences de l'éducation, 24(1), 3-29. https://www.erudit. org/fr/revues/rse/1998-v24-n1-rse1840/031959ar.pdf

Lowe, A. (2002). La pédagogie actualisante ouvre ses portes à l'interdisciplinarité scolaire. Éducation et francophonie, 30(2), 220-240. http://collections.banq.qc.ca/ ark:/52327/bs61543

Luquette, M. (2017). Nature et rôle des inférences impliquées dans la résolution de problèmes mathématiques [Thèse de doctorat, Université de Montréal]. Papyrus. http://hdl.handle.net/1866/20053

Magnan, C.-J. et Ahern, J. (1922). Mon premier livre : lire, écrire, compter. ÉducationInstruction, manuel des commerçants (Première partie). Conseil de l'instruction publique, Gouvernement du Québec.

Ministère de l'Éducation du Québec [MEQ]. (2000). Programme de formation de l'école québécoise (version préliminaire du programme du primaire, 00-0439). Gouvernement du Québec.

Ministère de l'Éducation du Québec [MEQ]. (2006). Programme de formation de l'école québécoise (version approuvée). Gouvernement du Québec. http://www. education.gouv.qc.ca/enseignants/pfeq/primaire 
Morand, B. (2020). Étude des structurations du contrôle déployées lors de la résolution en équipe de problèmes algébriques [Mémoire de maîtrise, Université du Québec à Montréal]. Archipel. http://archipel.uqam.ca/id/eprint/13933

Mottier Lopez, L. (2007). Régulations interactives situées dans des dynamiques de microculture de classe. Mesure et Évaluation en Éducation, 30(2), 23-47. https:// www.researchgate.net/publication/280305760_Regulations_interactives_situees dans_des_dynamiques_de_microculture de_classe

Perkins, D. N. et Simmons, R. (1988). Patterns of misunderstanding: An integrative model for science, math, and programming. Review of Educational Research, 58(3), 303-326. https://doi.org/10.3102/00346543058003303

Radford, L. (1996). Sur la résolution de problèmes : comprendre puis résoudre. Bulletin de l'AMQ, 36(3), 19-30. http://www.luisradford.ca/ pub/108_1996AMQLaresolutiondeproblemesSv_2.pdf

Reusser, K. (1990). From text to situation to equation: cognitive simulation of understanding and solving mathematical word problems. Dans H. Mandl, E. De Corte, S. N. Bennett et H. F. Friedrich (dir.), Learning \& instruction: European research in an international context (vol. 2, p. 477-498). Pergamon Press.

Rhéaume, S. (2020). Les prises de décision des élèves du 3e cycle du primaire lors de la résolution de problèmes de proportion : une analyse des contrôles mobilisés [Thèse de doctorat, Université Laval]. CorpusUL. http://hdl.handle. $\underline{\text { net } / 20.500 .11794 / 40080}$

Richard, J.-F. (1995). Les activités mentales. Comprendre, raisonner, trouver des solutions. Armand Colin.

Saboya, M. (2010). Élaboration et analyse d'une intervention didactique co-construite entre chercheur et enseignant, visant le développement d'un contrôle sur l'activité mathématique chez les élèves du secondaire. [Thèse de doctorat, Université du Québec à Montréal]. Archipel. https://archipel.uqam.ca/3107/1/D1933.pdf

Saboya, M. et Rhéaume, S. (2015). Quel contrôle exercent les élèves lors de la résolution d'un problème de comparaison de fractions ? Petit x, (99), 5-31. https://www. researchgate.net/publication/291354708 
Saboya, M. et Tremblay, M. (2017). Co-élaboration d'interventions entre enseignantes et chercheures visant le développement d'un choix éclairé de matériel auprès d'élèves en difficulté d'apprentissage au primaire dans la résolution de problèmes additifs. Dans A. Braconne-Michoux, P. Gibel et I. Oliveira (dir.), Étude de différentes formes d'interactions entre recherches en didactique des mathématiques et formations professionnelles des enseignants. Livres en ligne du CRIRES. http://lel.crires.ulaval.ca/public/BraconneMichoux_Gibel Oliveira_2017.pdf

Saboya, M. et Tremblay, M. (2019, 24-26 mai). Le travail mené en français comme support à la résolution d'histoires mathématiques dans des classes de $2 \mathrm{e}$ et $3 \mathrm{e}$ années en adaptation scolaire. Dans C. Corriveau, V. Martin, M. Thibeault et A. Savard (dir.), Actes du colloque GDM2019. À quoi ressemble aujourd'hui la recherche en didactique des mathématiques au Québec? (p. 251-258). Université Laval. https://www.gdm.quebec/colloque/actes

Saboya, M., Bednarz, N. et Hitt, F. (2015). Le contrôle exercé en algèbre : conceptualisation et analyses en résolution de problèmes. Annales de didactique et de sciences cognitives, 20, 61-100. https://www.researchgate.net/ publication $/ 281904073$

Schleppegrell, M. J. (2007). The linguistic challenges of mathematics teaching and learning: A research review. Reading \& Writing Quarterly, 23(2), 139-159. https://doi.org/10.1080/10573560601158461

Søvik, N., Frostrad, P. et Heggberget, M. (1999). The relation between reading comprehension and task-specific strategies used in arithmetical word problems. Scandinavian Journal of Educational Research, 43(4), 371-398. https://doi. org/10.1080/0031383990430403

Thyrion, F. (1997). L'écrit argumenté. Questions d'apprentissage. Peeters.

Tremblay, M. et Dumas, B. (2011). Quand interroger l'activité mathématique à privilégier dans la classe contribue à développer les compétences. Vie pédagogique, (160), 64-69. https://collections.banq.qc.ca/ark:/52327/bs2101109

Vergnaud, G. (1982). A classification of cognitive tasks and operations of thought involved in addition and subtraction problem. Dans T. P. Carpenter, J. M. Moser, 
T. A. Romberg (dir.), Addition and Subtraction: A cognitive perspective (chap. 4). Lawrence Erlbaum.

Vilenius-Tuohimaa, P. M., Aunola, K. et Nurmi, J.-E. (2008). The association between mathematical word problems and reading comprehension. Educational Psychology, 28(4), 409-426. https://doi.org/10.1080/01443410701708228

Voyer, D., Beaudoin, I. et Goulet, M.-P. (2012). De la lecture à la résolution de problèmes : des habiletés spécifiques à développer. Revue canadienne de l'éducation, 35(2), 401-421. https://journals.sfu.ca/cje/index.php/cje-rce/article/view/1290

Williams, J. P. (2003). Teaching text structure to improve reading comprehension. Dans H. L. Swanson, K. Harris et S. Graham (dir.), Handbook of learning disabilities (1re éd., p. 295-305). Guilford Press.

Williams, J. P. (2005). Instruction in reading comprehension for primary grade students: A focus on text structure. Journal of Special Education, 39(1), 6-18. https://doi. org/10.1177/00224669050390010201

Williams, J. P., Hall, K. M. et Lauer, K. D. (2004). Teaching expository text structure to young at-risk learners: Building the basics of comprehension instruction. Exceptionality, 12(3), 129-144. https://doi.org/10.1207/s15327035ex1203_2

Williams, J. P., Hall, K. M., Lauer, K. D., Stafford, K. B., De Sisto, L. A. et deCani, J. S. (2005). Expository text comprehension in the primary grade classroom. Journal of Educational Psychology, 97(4), 538-550. https://doi. org/10.1037/0022-0663.97.4.538

Williams, J. P., Nubla-Kung, A. M., Pollini, S., Stafford, K. B., Garcia, A. et Snyder, A. E. (2007). Teaching cause-effect structure through social studies content to at-risk second graders. Journal of Learning Disabilities, 40(2), 111-120. https://doi.org/1 $\underline{0.1177 / 00222194070400020201}$

Zakhartchouk, J.-M. (2001). Justifiez, expliquez... . Pratiques, (111-112), 179-188. https://doi.org/10.3406/prati.2001.2430 